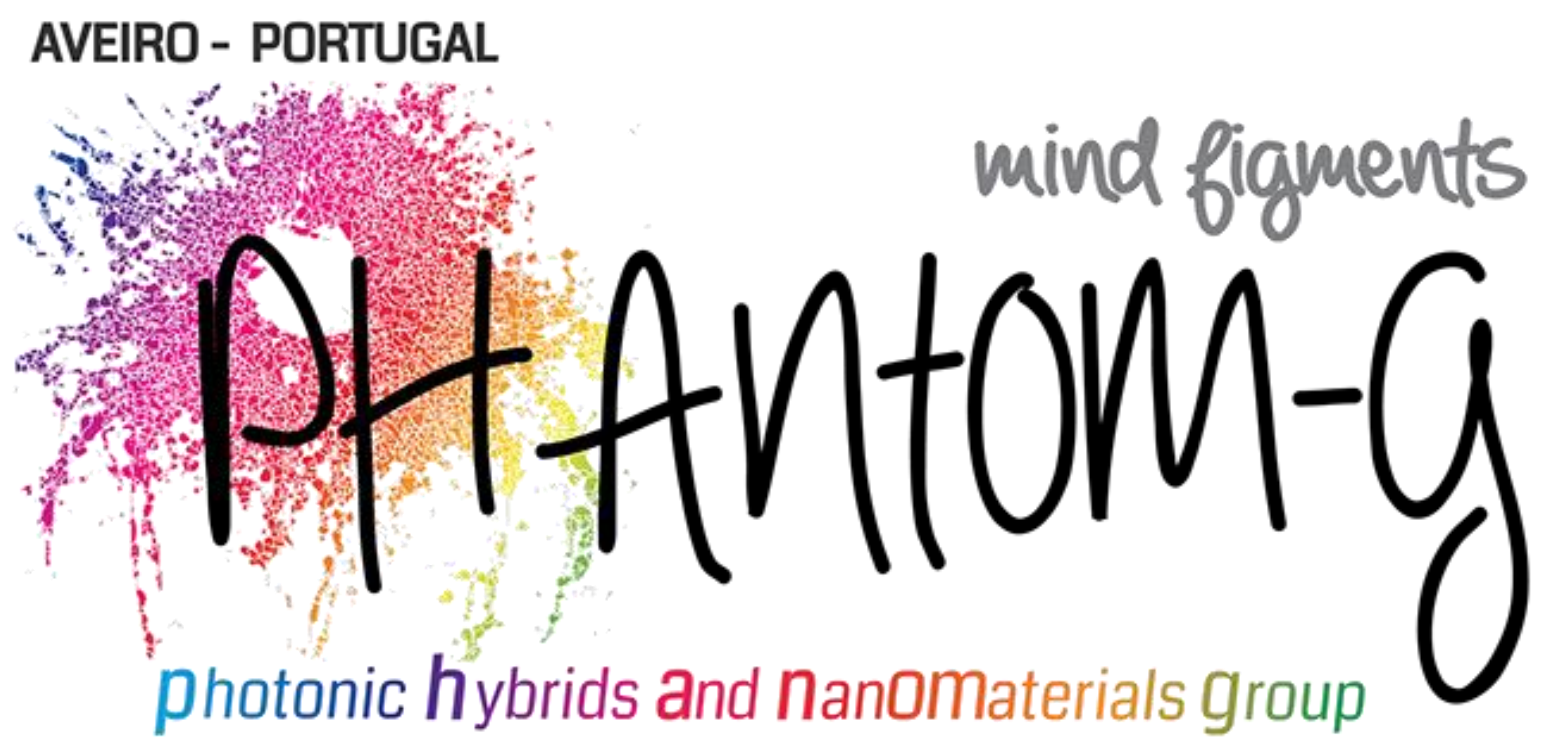

Please cite this article as: Hernández-Rodríguez, M. A., Brites, C. D. S., Antorrena, G., Piñol, R., Cases, R., Pérez-García, L., Rodrigues, M., Plaza, J. A., Torras, N., Díez, I., Millán, A., Carlos, L. D., Lanthanide Luminescence to Mimic Molecular Logic and Computing through Physical Inputs. Adv. Optical Mater. 2020, 2000312. https://doi.org/10.1002/adom.202000312 


\section{Lanthanide Luminescence to Mimic Molecular Logic and Computing through Physical Inputs}

Miguel A. Hernández-Rodríguez, Carlos D. S. Brites", Guillermo Antorrena, Rafael Piñol, Rafael Cases, Lluïsa Pérez-García, Mafalda Rodrigues, José António Plaza, Nuria Torras, Isabel Díez, Angel Millán and Luís D. Carlos*

Dr. Miguel A. Hernández-Rodríguez, Prof. Carlos D. S. Brites, Prof. Luís D. Carlos Phantom-g, CICECO - Aveiro Institute of Materials, Department of Physics, Universidade de Aveiro 3810-193 Aveiro, Portugal

E-mail: carlos.brites@ua.pt, lcarlos@ua.pt

Dr. Guillermo Antorrena

Laboratório de Microscopías Avanzadas (LMA), Instituto de Nanociencia de Aragón (INA) Universidad de Zaragoza, Edificio I+D, C./Mariano Esquillor s/n., 50018 Zaragoza Spain

Dr. Rafael Piñol, Prof. Rafael Cases, Dr. Angel Millán

Departamento de Física de la Materia Condensada, and Instituto de Ciencia de Materiales de Aragón, CSIC-Universidad de Zaragoza, 50009 Zaragoza

Spain

Prof. Lluïsa Pérez-García, Dr. Mafalda Rodrigues

Department de Farmacologia i Química Terapèutica and Institut de Nanociència i

Nanotecnologia UB (IN2UB), Universitat de Barcelona, Avda. Joan XXIII s/n, 08028

Barcelona

Spain

Prof. José António Plaza, Dr. Nuria Torras

Instituto de Microelectrónica de Barcelona, IMB-CNM (CSIC), Campus UAB, 08193

Bellaterra, Barcelona

Spain

Dr. Isabel Díez

Department of Applied Physics

Aalto University Helsinki

Finland

Keywords: molecular logic, computing, physical input, lanthanide, luminescence 


\begin{abstract}
The remarkable advances in molecular logic reported in the last decade demonstrated the potential of luminescent molecules for logical operations, a paradigm-changing concerning silicon-based electronics. Trivalent lanthanide $\left(\mathrm{Ln}^{3+}\right)$ ions, with their characteristic narrow line emissions, long-lived excited states, and photostability under illumination, may improve the state-of-the-art of molecular logical devices. Here is reported the use of monolithic siliconbased structures incorporating $\mathrm{Ln}^{3+}$ complexes for performing logical functions. Elementary logic gates (AND, INH and DEMUX), sequential logic (KEYPAD LOCK), and arithmetic operations (HALF ADDER and HALF SUBTRACTOR) exhibiting a switching ratio >60\% are demonstrated for the first time using non-wet conditions. Additionally, this is the first report showing sequential logic and arithmetic operations combining molecular $\mathrm{Ln}^{3+}$ complexes and physical inputs. Contrary to chemical inputs, physical inputs may enable the future concatenation of distinct logical functions and reuse of the logical devices, a clear step-forward towards input-output homogeneity that is precluding the integration of nowadays molecular logic devices.
\end{abstract}




\section{Introduction}

In a period of a few decades, the computers and related systems become smaller and smaller due to the substantial scaling shrinkage of silicon-based technology components. ${ }^{[1-5]}$ Whereas the first field-effect transistor has the dimensions of a palm of a hand, nowadays over 100 million equivalent components per $\mathrm{mm}^{2}$ are packaged in a chip using state-of-the-art $10 \mathrm{~nm}$ lithography. ${ }^{[6]}$ Nevertheless, the increasing demand for further computing power and downscaling are not compatible with the inherent scale limitation of lithographic methods, ${ }^{[7,8]}$ precluding much further miniaturization using the top-down approach followed so far. A disruptive step-forward towards bottom-up processing ${ }^{[9,10]}$ will be perhaps the only possibility to expand the current computing power overcoming the inherent limitations of nowadays electronic devices.

In the early 90's, Prasanna de Silva et al. ${ }^{[1]}$ described the first general and practical approach to information processing and computing based on molecules able to perform logical operations. The first AND molecular logic gate was based on organic molecules, applying cations as the logical inputs and a change in the emission intensity of the organic molecules as the logical output. ${ }^{[1]}$ Basic logical gates were the first generation of logic devices in the 2000s. ${ }^{[2,3,7,11-17]}$ Interrogating the molecules with ionic species, their optical properties were changed, ${ }^{[17-19]}$ giving a TRUE or FALSE logical output (logical outputs 1 and 0), and thus elementary Boolean functions were performed. Later on, it was recognized that the molecules can additionally perform logical operations of higher complexity, such as molecular multiplexers and demultiplexers or molecular binary adders and subtractors ${ }^{[18,20-25]}$ All the above-mentioned molecular devices operate in wet conditions, so its immediate use was in biomedicine. Molecular and biomolecular computing/logic systems ${ }^{[26-28]}$, including supramolecules, ${ }^{[16]}$ proteins, ${ }^{[14]} \mathrm{DNA}^{\left[{ }^{[29]} \mathrm{DNA} / \text { conjugated polymer systems, }\right.}{ }^{[17,21]}$ and enzyme based-systems ${ }^{[30]}$, were used to process chemical input signals mimicking most of the logical operations of 
As the signal processing is performed by molecules, parallel computing can be implemented, allowing an exponential gain on computing power in comparison with nowadays electronic devices. ${ }^{[31]}$ However, the characteristic broadband emission and fast decay times of organic compounds, conjugated to the well-known photobleaching, precludes multiplexing using light as a logical input or output, making difficult its implementation in applications other than proof of concept molecular logic devices.

The use of trivalent lanthanide ions $\left(\mathrm{Ln}^{3+}\right)$ as luminescent optical active centers in molecular logic is particularly attractive as these ions typically emit in a wide wavelength range covering the UV-VIS-NIR spectral regions, with characteristic line-like emission bands $(<10 \mathrm{~nm})$ and long-lived excited state lifetimes ( $>1 \mu \mathrm{s})$. Despite it is nowadays recognized that $\mathrm{Ln}^{3+}$ ions can improve the state-of-the-art of molecular logical devices, only a handful of papers have been published so far. ${ }^{[11,32-36]} \mathrm{Up}$ to now, all the logical gates based on $\mathrm{Ln}^{3+}$ ions respond to chemical inputs and thus operate exclusively in wet conditions, except the $\mathrm{Eu}^{3+} / \mathrm{Tb}^{3+}$ based selfassembled polymer monolayer functionalized Si surface proposed by some of us in 2016. ${ }^{[36]}$

It is recognized in the literature that if physical inputs (viz. light, pressure, temperature) are used as inputs and outputs of a molecular logical device it will render advantages in the inputoutput homogeneity when the integration of distinct logical functions, no-contamination, and reuse of the device are demanded. ${ }^{[37]}$ It also permits higher integration of distinct logical functions because it does not require physical access, and monolithic structures may contain thousands of individual logic elements. For example, the optical inputs signals can be remotely delivered and read from the molecular devices as time-gated pulses (covering time ranges until the picosecond, ${ }^{[37]}$ faster cycling even in comparison to the electronic counterparts). All these potential benefits motivate the transfer of molecular logic devices from wet (chemical or physical inputs and physical outputs) to dry (exclusively physical inputs and outputs) operation conditions. Up to now all the works performing molecular logic and computing using 
exclusively physical inputs (involving reversible photo transformations of photochromic molecules $)^{[37-41]}$ operate, however, in wet conditions.

Here, we report a noteworthy step forward relative to the state-of-the-art examples presenting high-sophistication-level logical operations based exclusively on physical inputs and outputs and operating in non-wet conditions. By exploiting a $\mathrm{Eu}^{3+} / \mathrm{Tb}^{3+}$-functionalized $\mathrm{Si}$ platform, that produces narrow and stable emission lines and that is fully compatible with existent Si-based electronic devices, we fabricate molecular logical arrays performing basic (AND, INH, DEMUX), sequential (KEYPAD LOCK), and arithmetic (HALF ADDER and HALF SUBTRACTOR) logic operations.

\section{Results and Discussion}

\subsection{Fabrication and Structural Characterization of the Logical Arrays}

The formation and the structure of the $\mathrm{Eu}^{3+} / \mathrm{Tb}^{3+}$ based self-assembled polymer monolayer functionalized Si surface are depicted in Figure 1a, and Figure 1b respectively. Original monocrystalline $\mathrm{Si}$ wafers were covered with a $1 \mu \mathrm{m}$ thick layer of $\mathrm{SiO}_{2}$ and the surface was activated by amination with 3-aminopropyl triethoxysilane (APTES). The functionalization with the $\mathrm{Eu}^{3+}$ and $\mathrm{Tb}^{3+}$ complexes was realized by Michael's addition to the surface amine groups of an acrylate poly(ethylene glycol) (PEG) polymer spacer terminated in a $\beta$-ketoester ligand (Figure S1 and Table S1 in Supporting Information). This group fixes the $\mathrm{Eu}^{3+}$ and $\mathrm{Tb}^{3+}$ ions to the surface allowing further complexation of these ions with light-harvesting DPA $(2,6-$ piridindicarboxilic acid) ligands. Different types of coatings were prepared that differ in the length of the PEG polymer spacer (Figure 1c, Table 1). Samples with and without the addition of the $\mathrm{Ln}^{3+}(\mathrm{Ln}=\mathrm{Eu}, \mathrm{Tb})$ ions and DPA ligands and using two different reaction times for Michael addition of acrylate to surface amines were prepared (Figures S2-S4 and Tables S2-S4 in Supporting Information). 
The extension of the reaction time from $24 \mathrm{~h}$ (samples S1B-S6B) to $48 \mathrm{~h}$ (samples S1DS6D) did not result in a significant change in the composition and structure of the samples, and therefore samples of the series B and D are equivalent from the luminescence and structural point-of-view. The same applies to the samples without $\mathrm{Eu}^{3+}$ and $\mathrm{Tb}^{3+}$ ions prepared with a reaction time of $24 \mathrm{~h}$ (samples S1A-S6A) and $48 \mathrm{~h}$ (samples S1C-S6C).

The samples were analyzed by X-ray photoelectron spectroscopy (XPS) before and after the formation of the $\mathrm{Eu}^{3+}$ and $\mathrm{Tb}^{3+}$ complexes, confirming the functionalization of the Si surface and the incorporation of the $\mathrm{Ln}^{3+}$ ions (Figure 2)

Field emission scanning electron microscopy analysis of the samples show a very smooth surface and a uniform chemical composition for the samples with medium and small polymer chain lengths, whereas some degree of granulation and chemical heterogeneity is discerned for the sample with the longest polymer length. Atomic force microscopy analysis (Figures S5-S8 in Supporting Information) shows a clear increase of the surface roughness on the $\mathrm{Ln}^{3+}$ functionalized samples concerning amine-modified samples. This loss of functionalization effectiveness in long polymeric chain lengths is also observed in the $\mathrm{Ln}^{3+}$-doped samples (S1B to $\mathbf{S 6 B}$ ), and it is reflected in a lower concentration of $\mathrm{Eu}^{3+}$ and $\mathrm{Tb}^{3+}$ on the surface (Tables S2 and S3 in Supporting Information).

\subsection{Photoluminescence}

The photoluminescence of the samples prepared with distinct acacPEGA chain lengths or with different functionalization routes do not show notable differences (Figures S10-S17 in Supporting Information) and, thus, we selected for further investigation three representative samples treated at $48 \mathrm{~h}$ with the longest (S1D), middle (S3D) and shortest (S6D) polymer chain length.

The room temperature excitation spectra of the three samples, monitoring the ${ }^{5} \mathrm{D}_{4} \rightarrow{ }^{7} \mathrm{~F}_{5}$ $\left(\mathrm{Tb}^{3+}\right)$ and ${ }^{5} \mathrm{D}_{0} \rightarrow{ }^{7} \mathrm{~F}_{2}\left(\mathrm{Eu}^{3+}\right)$ transitions, are similar and dominated by broadband in the $250-290$ 
$\mathrm{nm}$ range associated to the DPA ligand excited states (Figure S10 in Supporting Information). ${ }^{[42]}$ Increasing the temperature, the integrated areas of the ${ }^{5} \mathrm{D}_{4} \rightarrow{ }^{7} \mathrm{~F}_{5}\left(\mathrm{~Tb}^{3+}, I_{1}\right)$ and ${ }^{5} \mathrm{D}_{0} \rightarrow{ }^{7} \mathrm{~F}_{2}\left(\mathrm{Eu}^{3+}, I_{2}\right)$ transitions decrease, by 70 and $30 \%$ respectively (Figure 3 and Figure S12 in Supporting Information), meaning that the $I_{1} / I_{2}$ ratio is temperature-sensitive, as reported for other $\mathrm{Eu}^{3+} / \mathrm{Tb}^{3+}$-bearing materials. ${ }^{[43-47]}$ Figure S18 in Supporting Information presents the temperature evolution of the $I_{1} / I_{2}$ ratio in a single heating-cooling cycle for S1D, S3D, and S6D. During the heating from 295 to $369 \mathrm{~K}$, the intensity ratio decreases linearly, probably due to the activation of nonradiative decay pathways involving the polymeric chains that depopulate the ${ }^{5} \mathrm{D}_{4}$ and ${ }^{5} \mathrm{D}_{0}$ emitting levels. The high energy of the DPA ligand triplet state, $\sim 27050 \mathrm{~cm}^{-1},{ }^{[46}$, ${ }^{48]}$ prevents the ${ }^{5} \mathrm{D}_{4}$-to-ligand and ${ }^{5} \mathrm{D}_{0}$-to-ligand energy back-transfer, discarding, then, any role of the ion-to-ligand back transfer in the decrease of the $I_{1} / I_{2}$ ratio as temperature increases. Furthermore, thermally activated $\mathrm{Tb}^{3+}$-to-Eu ${ }^{3+}$ energy transfer is also settled out as no evidence of that transfer is found in the excitation spectra and lifetime measurements (in accord with the relatively low amount of the two ions, making the energy transfer extremely improbable). Moreover, the $I_{1} / I_{2}$ ratio shows a hysteretic behavior in a heating/cooling cycle (Figures S18 in Supporting Information). This is not related to the optical bistability of $\mathrm{Ln}^{3+}$-bearing crystals excited with high power densities, ${ }^{[49]}$ but to changes in the conformation of the acacPEGA polymer chains, as reported previously by some of us in an analogous polymer. ${ }^{[36,50]}$ On cooling, two linear regimes are distinctly discerned, whose intersection occurs at the same temperature for all the samples, $\left(T_{\mathrm{c}}=313 \pm 1 \mathrm{~K}\right)$ (Figure S18 in Supporting Information). This indicates that the two regimes are independent of the polymeric chain length and different functionalization routes used (Figures S13-S14 in Supporting Information), reaffirming the crucial role of the temperature dependence of the polymer conformation on the temperature dependence of the $I_{1} / I_{2}$ ratio. The hysteretic response of the samples will be used in 2.3 .2 to demonstrate sequential logical operations. 
The thermometric response of these systems was accessed and summarized in Table S6 and Figures S18-S20 in Supporting Information. The maximum relative thermal sensitivities are 2.84, 2.23 and $2.27 \% \mathrm{~K}^{-1}$ for S1D, S3D and S6D respectively. These values are high enough to consider the application of these samples as luminescent molecular temperature probes (Table 2), particularly in the physiological range of temperatures interesting for biological applications. However, the focus of this manuscript is to use $\mathrm{Ln}^{3+}$ luminescence, including its temperature dependence, to mimic molecular logic and computing through physical inputs.

\subsection{Molecular Logic Gates}

\subsubsection{Combinational Logic Functions}

The Si arrays functionalized with $\mathrm{Eu}^{3+}$ and $\mathrm{Tb}^{3+}$ ions emitting centers can be employed for the construction of optically active molecular logic gates, using exclusively physical inputs and outputs. For the following discussion, we use S1D as an illustrative example. We tackle the design of molecular logics exploiting the heat and the excitation wavelength as logical inputs and several emission intensity ratios as the logical outputs (Figure 3d,e). The excitation light is always assumed as an input in the reports on all-optical or all-photonic logic gates, ${ }^{[37,51-54]}$ however it is the first time that temperature increase (or heat) is being used as an input for molecular logical gates. Using simply the dependence of the emission spectra upon $271 \mathrm{~nm}$ excitation as the temperature is being raised (Figure 4a) we clear observe changes in the emission profile that can be quantified using three ratiometric outputs: (i) $I_{1} / I_{2}$, defined above, (ii) $I_{2 \mathrm{~A}} / I_{2 \mathrm{~B}}$, involving the intensity peak ratio of two Stark components of the ${ }^{5} \mathrm{D}_{0} \rightarrow{ }^{7} \mathrm{~F}_{2}$ transition, Figure $4 \mathrm{~b}$, and (iii) $I_{3 \mathrm{~A}} / I_{3 \mathrm{~B}}$, an intensity peak ratio of two Stark components of the ${ }^{5} \mathrm{D}_{0} \rightarrow{ }^{7} \mathrm{~F}_{4}$ transition Figure 4c. The temperature evolution of these intensity ratios is plotted in Figure 4d,e. Whereas $I_{1} / I_{2}$ and $I_{2 \mathrm{~A}} / I_{2 \mathrm{~B}}$ decrease 66 and $50 \%$, respectively, as temperature increases 70 degrees, for $I_{3 \mathrm{~A}} / I_{3 \mathrm{~B}}$ this same increment results in a $55 \%$ enhancement, relatively to the value at 
$300 \mathrm{~K}$. These dissimilar evolutions upon heating induced the development of one inhibit (INH) and one AND logical gates.

Using the $I_{1} / I_{2}$ ratio (or $I_{2 \mathrm{~A}} / I_{2 \mathrm{~B}}$ ) as logical output we set a threshold value of 0.06 to define the logical output TRUE (1, when $\left.I_{1} / I_{2}>0.06\right)$ or FALSE ( 0 , the opposite case). The logical inputs are the excitation at $271 \mathrm{~nm}$ (denoted by UV) and a temperature increase of 70 degrees (denoted by $\Delta T$ ). This logical gate does not produce any output without UV and thus the logical output is 0 . Only in the presence of excitation at $271 \mathrm{~nm}$ and in the absence of $\Delta T$ the logical output is 1 and, thus, this is the transfer function characterizing the INH logic gate (Figure $4 a, c, e)$. We notice the high obtained switching ratio (66\% of the initial value); defined as the relative change of the output signal when the logical output signals change from " 1 " and " 0 ".

The $I_{3 A} / I_{3 B}$ ratio is used as logical output setting a threshold value of 0.8 to define the logical output TRUE (1, when $\left.I_{3 \mathrm{~A}} I_{3 \mathrm{~B}}>0.8\right)$ or FALSE (0, the opposite case). The logical inputs are the same defined for the INH logic gate. Again, this logical gate does not produce any output without $271 \mathrm{~nm}$ excitation and thus the logical output is 0 . Only when the system is excited at $271 \mathrm{~nm}$ and heated by $\Delta T$ the logical output is 1 and, thus, this is the transfer function characterizing the AND logic gate (Figure 4b,d,f). This gate operates based on the distinct thermal population ratio of two ${ }^{7} \mathrm{~F}_{4}$ Stark components. We observe that on one hand, the integrated intensity of the high-energy ${ }^{7} \mathrm{~F}_{4}$ Stark component is almost temperature independent whereas that of the remaining ones displays a strong temperature dependence (Figure S17 in Supporting Information) evidencing the hypersensitive nature of the ${ }^{5} \mathrm{D}_{0} \rightarrow{ }^{7} \mathrm{~F}_{4} \mathrm{Eu}^{3+}$ transition. ${ }^{[55]}$ The switching ratio of this gate is about $60 \%$, a similar value to that calculated from the data in reference ${ }^{[56]}(67 \%)$.

Observing the inputs homogeneity on the two logical gates described above we design a port constituted by the integration of the AND and the INH logic gates. The resulting logical gate, presented in Figure 4g,h, is a 1:2 demultiplexer (DEMUX). This is a clear step forward relative 
to our previous work ${ }^{[36]}$ (the first example using $\mathrm{Eu}^{3+}$ and $\mathrm{Tb}^{3+}$ emissions and temperature for logic applications), that reported the same DEMUX using a much complicated and timeconsuming strategy.

\subsubsection{Sequential Logical Functions}

Contrarily to the combinational logic functions described in the previous section, in the sequential logic functions the output signal switches $\mathrm{ON}$ when a specific inputs order is set, ${ }^{[57-}$ ${ }^{59]}$ implying a memory function of the device. The KEYPAD LOCK function has been reported previously employing both chemical ${ }^{[58,60-62]}$ and physical ${ }^{[37,61,63-68]}$ inputs. Here, a KEYPAD LOCK was implemented using the temperature increment $\Delta T$ and the characteristic temperature $T_{\mathrm{c}}$ as physical inputs (Figure 5a). We set the logical value 0 for the $\Delta T$ input representing the switching off of the temperature controller and the $T_{\mathrm{c}}$ input in the $T<T_{\mathrm{c}}$ range. The logical value 1 is set for the switching on of the temperature controller and $T=T_{\mathrm{c}}$, respectively. Each input might be 0 or 1 , leading to eight possible two-digit order combinations. From this state, when both physical inputs present the logical value 0 , the output is strictly $\left(I_{1} / I_{2}\right)_{0}$. Regardless of the order in which the temperature controller is switched on $(\Delta T>0)$ while the second input is $T<T_{\mathrm{c}}$, the output will be $\left(I_{1} / I_{2}\right)_{\mathrm{c}}<\left(I_{1} / I_{2}\right)<\left(I_{1} / I_{2}\right)_{0}$. On the other hand, when the temperature is fixed to $T_{\mathrm{c}}$ and no heating source is acting (and vice-versa), the output will be $\left(I_{1} / I_{2}\right)_{\mathrm{c}}$. When the heating is on, and then $T=T_{\mathrm{c}}$, the output yields $\left(I_{1} / I_{2}\right)_{\mathrm{c}} \leq I_{1} / I_{2}<\left(I_{1} / I_{2}\right)_{0}$.

As the initial state, we consider the sample a room temperature (denoted by $\left(I_{1} / I_{2}\right)_{0}$, Figure 5a,c). The only possibility to open the KEYPAD LOCK is to have first the system at $T=T_{\mathrm{c}}$ and then impose a $\Delta T$ such that $I_{1} / I_{2}<\left(I_{1} / I_{2}\right)_{\mathrm{c}}$.

\subsubsection{Arithmetic Operations}

A key requirement of digital computers is the ability to use logical functions to perform arithmetic operations. Traditionally the operation is implemented using an exclusively or (XOR for the sum digit) and an AND logical gate (for the carry digit). We notice that using the $U V$ 
and $\Delta T$ inputs it is not possible to recover the XOR transfer function, so we implement the HALF-ADDER operation using the temperature increase $\Delta T=T_{\mathrm{H}}-T_{\mathrm{C}}$ (Figure $5 \mathrm{a}, \mathrm{b}$, logical value 1 means heating and 0 means no temperature increase) and the characteristic temperature value $T=T_{\mathrm{c}}$ as inputs, and combining the $I_{1} / I_{2}$ output and the relative thermal sensitivity, defined by Equation (1).

Defining the logical value " 1 " for $I_{1} / I_{2}$ ratio lower than the threshold value $\left(I_{1} / I_{2}\right)_{\mathrm{H}}$ we recall the AND logic gate (for S1D $\left.\left(I_{1} / I_{2}\right)_{\mathrm{H}}=0.025\right)$. The $\left(I_{1} / I_{2}\right)_{\mathrm{H}}$ is defined as the ratio of integrated intensities on cooling corresponding to the temperature $T_{\mathrm{C}}$. The temperature $T_{\mathrm{H}}$ is defined as the temperature value for the heating regime for which the $\left(I_{1} / I_{2}\right)_{\mathrm{H}}$ is obtained, Figure 5a. For the XOR logic gate we use the $S_{\mathrm{r}}$ output defining the logical value " 1 " when $S_{\mathrm{r}}$ is in the range $S_{\mathrm{C}}<S_{\mathrm{r}}<S_{\mathrm{H}}$ corresponding to the $S_{\mathrm{r}}$ values at room temperature and at $T_{\mathrm{H}}$. The resulting HALFADDER logical gates arrangement is presented in Figure 5e and the corresponding truth table in Figure 5f.

Other operations can be implemented by simply playing with the logic gates arrangement. Replacing the AND gate by an INH gate a HALF-SUBTRACTOR is implemented (see Figure $5 \mathrm{~g}, \mathrm{~h})$. In this case, the arithmetic difference between two binary numbers is given by the difference digit that corresponds to the XOR logic gate and the borrow that is given by an INH gate. To generate the INH gate we simply take the first logical output as the $I_{1} / I_{2}=\left(I_{1} / I_{2}\right)_{\mathrm{c}}$ (Figure S21 in Supporting Information).

\section{Conclusions}

In summary, monolithic structures consisting of Si surfaces functionalized with acacPEGA polymeric chains of controllable length and functionalized with $\mathrm{Eu}^{3+}$ and $\mathrm{Tb}^{3+}$ ions are reported for applications in molecular logic and computing using exclusively physical inputs. Basic (AND, INH and DEMUX), sequential (KEYPAD LOCK) and arithmetic (HALF ADDER and HALF SUBTRACTOR) logic operations are demonstrated using the excitation light and the 
temperature as inputs and ratios of emission intensities and the relative thermal sensitivity as outputs. The system is fully reversible containing $\sim 10^{6}$ emitting centers per $\mu \mathrm{m}^{2}$. Comparing the integration density of the $\mathrm{Eu}^{3+} / \mathrm{Tb}^{3+}$ based self-assembled polymer monolayer functionalized Si surface with that of the modern Si-based chips $\left(\sim 10^{2}\right)^{[6]}$, we realize that the former presents $10^{4}$ times higher value. Concerning the molecular logical devices reported so far, this work demonstrates clear advantages on the integration of distinct logical functions and reuse of the device for several cycles. In this work, there is no physical contact of the inputs with samples (like in those operating in wet conditions) that result eventually in the device contamination. The implementation of the reported logical functions on a functionalized Sichip also permits better integration with current electronic devices and the use of $\mathrm{Ln}^{3+}$-based emitting centers is quite suitable for emission and excitation by commercial detectors and LED sources. Although a molecular computer able of substituting current conventional computers is still just an ambitious future vision, ${ }^{[24]}$ judiciously designing molecular systems that perform logic functions is paving the way for developing useful molecular logic applications. Most of the drawbacks of the devices reported so far can be mitigated if solid samples interrogated by physical inputs are used, preferentially giving narrow emitting lines. In this context, $\mathrm{Ln}^{3+}$-based emitting centers will certainly play a central role, since the same ions are widely spread in optoelectronic devices, smoothing the interface between novel molecular and conventional electronic devices.

\section{Experimental Section}

Materials: Europium(III) chloride hexahydrate $\left(\mathrm{EuCl}_{3}\left(\mathrm{H}_{2} \mathrm{O}\right)_{6}, 99.9 \%\right)$, Terbium(III) chloride hexahydrate $\left(\mathrm{TbCl}_{3}\left(\mathrm{H}_{2} \mathrm{O}\right)_{6}, 99.9 \%\right), 2,6$-Pyridinedicarboxylic acid (DPA, 99\%), tert-butyl acetoacetate, APTES, Diethyleneglycol (DEG), PEGX (where X=200, 300, 400, 600, 1000 and $3000 \mathrm{Da}$ is the $\mathrm{Mw}$ molecular weight), N,N-dimethylaniline (DMA), and acryloyl chloride (97\%) were purchased from Aldrich and used as received. 
Preparation of $\mathrm{SiO}_{2}$ coated wafers: p-type monocrystalline $\mathrm{Si}$ wafers (Okmetic) were coated with a $\mathrm{SiO}_{2}$ layer of $1083.0 \pm 11.3 \mathrm{~nm}$ thickness by Plasma-Enhanced Chemical Vapor Deposition of TEOS (tetraethylorthosilicate) at $380^{\circ} \mathrm{C}$, and a pressure of $1 \mathrm{~atm}$.

Surface activation: The silicon wafers were cut in $1 \times 1 \mathrm{~cm}^{2}$ dices, washed with ethanol and acetone and dried under nitrogen. Surface hydroxylation was performed by $\mathrm{O}_{3}$ treatment for 10 minutes using a UVO-cleaner Model 342 (Jelight Company Inc., USA).

Amine surface functionalization: Functionalization with amine groups was achieved by sol-gel reaction with APTES. The APTES coating was carried out by vapor deposition, as described in our previous publication. ${ }^{[36]}$ Briefly, the surfaces together with a vial containing $1 \mathrm{~mL}$ of APTES were placed inside a desiccator previously thermostated at $80^{\circ} \mathrm{C}$ and connected to a vacuum. The desiccator was pumped down and sealed off. The samples were left in contact with the APTES vapor for 3 hours. Afterward, the samples were washed with acetone, dried under nitrogen, and cured in an oven at $50-60^{\circ} \mathrm{C}$ for 20 minutes.

Grafting PEG polymer chains to the silicon substrates: The PEG polymer chains grafting were achieved by Michael's reaction of acrylate acacPEGA terminal groups with surface amine groups. After coating with APTES, the silicon surfaces were immersed in acacPEGA solution (samples $\mathbf{1 b}-\mathbf{6 b}, 8 \mathrm{mM}$, Supporting Information) in the water at $\mathrm{pH}=8.5$ and allowed to react at $60^{\circ} \mathrm{C}$, washed with distilled water and dried under nitrogen flux. The reaction was carried out individually for each wafer in an Eppendorf containing $2 \mathrm{~mL}$ of an $8.9 \mathrm{mM}$ aqueous solution of the polymer. Two series of silicon substrates with grafted polymer chains were prepared, labeled as S1A-S6A for a Michael reaction time of 24h and as S1C-S6C, for a Michael reaction time of $48 \mathrm{~h}$ (Table 1$)$.

Anchoring of lanthanide coordination compounds: Europium and terbium ions in a 1:3 molar ratio were attached to the polymer self-assembled monolayer by coordination to the $\beta$-ketoester polymer terminal groups in a $1: 1$ polymer $/ \mathrm{Ln}^{3+}$ ratio. The polymer-coated wafers were placed in a vial with $2 \mathrm{~mL}$ of water, to which it was added $15.8 \mu \mathrm{L}$ of $\mathrm{NaOH} 1 \mathrm{M}, 100 \mu \mathrm{L}$ of a solution of $\mathrm{EuCl}_{3}\left(\mathrm{H}_{2} \mathrm{O}\right)_{6}(1.44 \%$ w. $)$ and $100 \mu \mathrm{L}$ of a solution of $\mathrm{TbCl}_{3}\left(\mathrm{H}_{2} \mathrm{O}\right)_{6}(4.42 \%$ w. $)$, and the $\mathrm{pH}$ was adjusted to 6.7 . After 3 hours, $340 \mu \mathrm{L}$ of a solution of DPA $(15.75 \mathrm{mg} / \mathrm{mL}$, pH 6.76$)$ was added. The complex was formed overnight. The surfaces were then thoroughly washed with distilled water and dried under nitrogen. Two series of samples were obtained, samples S1BS6B (deriving from samples S1A-S6A), and samples S1D-S6D (deriving from samples S1CS6C) (Table 1).

Surface characterization by XPS: The X-ray photoelectron spectroscopy (XPS) analysis was carried out using a Kratos Axis Ultra spectrometer employing a monochromatic Al Ka $(1486,6$ eV) $10 \mathrm{~mA}, 15 \mathrm{kV}$ ) X-ray source and a power of $150 \mathrm{~W}$. All samples were introduced in the analysis chamber simultaneously and were analyzed in the same experimental conditions. To avoid any induced effect in the chemistry of the samples, they were analyzed as received without any previous etching. Differential surface charging was minimized using a charge neutralizer system (flood gun). Survey spectra were recorded using an analyzer pass energy of $160 \mathrm{eV}$ and $1.0 \mathrm{eV}$ energy step. High-resolution spectra of C 1s, O 1s, N 1s, Si 2p, Eu 3d, and $\mathrm{Tb} 3 \mathrm{~d}$ regions were collected using pass energy of $20 \mathrm{eV} 0.1 \mathrm{eV}$ energy step. The spectra were analyzed using CasaXPS ${ }^{\circledR}$ software. The background for all spectra was subtracted using a Shirley baseline. Due to the use of charge neutralizer, spectra need to be calibrated. Binding Energies (B.E.) were referenced to the $\mathrm{C} 1 \mathrm{~s}(\mathrm{C}-\mathrm{C})$ peak at $285.0 \mathrm{eV}$. 
Photoluminescence characterization: The emission spectra were recorded with a modular double grating excitation spectrofluorimeter with a TRIAX 320 emission monochromator (Fluorolog-3. Horiba Scientific) coupled to an R928 Hamamatsu photomultiplier, using a front face configuration. A $450 \mathrm{~W}$ Xe arc lamp was used as the excitation source. Both recorded emission and excitation spectra were corrected with the spectrofluorimeter optical spectral response and the spectral distribution of the lamp intensity using a photodiode reference detector respectively. On the other hand, the temperature was controlled using an IES-RD31 controller and a Kapton thermofoil heater from Minco mounted on a copper holder and monitored using a thermocouple thermometer Barnant 100 (model 600-2820) with an accuracy of $0.1 \mathrm{~K}$, accordingly to the manufacturer. The temperature ramp in the heating-cooling cycles is about $0.25 \mathrm{~K} \mathrm{~min}^{-1}$.

Thermometric performance: The relative thermal sensitivity $\left(S_{\mathrm{r}}\right)$ was calculated using:

$$
S_{r}=\frac{1}{\Delta}\left|\frac{\partial \Delta}{\partial T}\right| .
$$

and the temperature uncertainty using:

$$
\delta T=\frac{1}{S_{r}} \frac{\delta \Delta}{\Delta} .
$$

being $\delta \Delta \Delta$ the relative uncertainty in $\Delta$ calculated using:

$$
\delta \Delta=\sqrt{\left(\frac{\delta I_{1}}{I_{1}}\right)^{2}+\left(\frac{\delta I_{2}}{I_{2}}\right)^{2}}
$$

where $\delta I_{i} / I_{i}(i=1.2)$ is calculated dividing the readout fluctuations of the baseline by the maximum value of each intensity, i.e. $I_{l}$ and $I_{2}$. As the integrated areas are calculated from the same emission spectra $\delta I_{l}=\delta I_{2}=\delta I$. The experimental $\Delta=I_{\mathrm{Tb}} / I_{\mathrm{Eu}}$ values were fitted to straight lines (Table S6 in Supporting Information) and the resulting parameters were used to calculate $S_{\mathrm{r}}$ and $\delta T$.

\section{Supporting Information}

Supporting Information is available from the Wiley Online Library or from the authors.

\section{Acknowledgments}

MAHR and CDSB contributed equally to this work. This work was developed within the scope of the project CICECO-Aveiro Institute of Materials, UIDB/50011/2020 \& UIDP/50011/2020, financed by national funds through the Portuguese Foundation for Science and Technology/MCTES. This work was financially supported by the project NanoHeatControl, (POCI-01-0145-FEDER-031469), funded by FEDER, through POCI, and by Portuguese funds (OE), through FCT/MCTES, and by the Spanish Ministry of Science Innovation and Universities (Grant No.: PGC2018_095795_B_I00) and the Diputación General de Aragón (Grant No.: E11/17R). CDSB and MAHR thank SusPhotoSolutions (CENTRO-01-0145FEDER-000005) for grants.

Received: ((will be filled in by the editorial staff))

Revised: ((will be filled in by the editorial staff)) Published online: ((will be filled in by the editorial staff)) 


\section{References}

[1] A. P. de Silva, N. H. Q. Gunaratne, C. P. McCoy, Nature 1993, 364, 42.

[2] A. P. de Silva, S. Uchiyama, Nat. Nanotechnol. 2007, 2, 399.

[3] A. P. de Silva, Nature 2008, 454, 417.

[4] T. Baehr-Jones, T. Pinguet, P. Lo Guo-Qiang, S. Danziger, D. Prather, M. Hochberg, Nat. Photonics 2012, 6, 206.

[5] B. Daly, T. S. Moody, A. J. M. Huxley, C. Y. Yao, B. Schazmann, A. Alves-Areias, J. F. Malone, H. Q. N. Gunaratne, P. Nockemann, A. P. de Silva, Nat. Commun. 2019, 10, 49.

[6] J. C. Norman, D. Jung, Y. T. Wan, J. E. Bowers, APL Photonics 2018, 3, 030901.

[7] J. M. Tour, Acc. Chem. Res. 2000, 33, 791.

[8] L. Sun, Y. A. Diaz-Fernandez, T. A. Gschneidtner, F. Westerlund, S. Lara-Avila, K. Moth-Poulsen, Chem. Soc. Rev. 2014, 43, 7378.

[9] J. M. Tour, Chem. Rev. 1996, 96, 537.

[10] K. Szacilowski, Infochemistry: information processing at the nanoscale, John Wiley \& Sons, United Kingdom 2012.

[11] T. Gunnlaugsson, D. a. M. Dónail, D. Parker, Chem. Commun. $2000,93$.

[12] M. S. Tremblay, Q. Zhu, A. A. Marti, J. Dyer, M. Halim, S. Jockusch, N. J. Turro, D. Sames, Org. Lett. 2006, 8, 2723.

[13] A. P. de Silva, T. P. Vance, M. E. S. West, G. D. Wright, Org. Biomol. Chem. 2008, 6, 2468.

[14] P. L. Gentili, J. Phys. Chem. A 2008, 112, 11992.

[15] A. P. de Silva, C. M. Dobbin, T. P. Vancea, B. Wannalerse, Chem. Commun. 2009, 1386.

[16] H. Komatsu, S. Matsumoto, S. i. Tamaru, K. Kaneko, M. Ikeda, I. Hamachi, J. Am. Chem. Soc. 2009, 131, 5580.

[17] M. Moshe, J. Elbaz, I. Willner, Nano Lett. 2009, 9, 1196.

[18] F. Pu, E. Ju, J. Ren, X. Qu, Adv. Mater. 2014, 26, 1111.

[19] R. Arabahmadi, Talanta 2019, 194, 119.

[20] S. Erbas-Cakmak, S. Kolemen, A. C. Sedgwick, T. Gunnlaugsson, T. D. James, J. Yoon, E. U. Akkaya, Chem. Soc. Rev. 2018, 47, 2228.

[21] F. Pu, C. Wang, D. Hu, Z. Huang, J. Ren, S. Wang, X. Qu, Mol. BioSyst. 2010, 6, 1928.

[22] A. Prasanna De Silva, Chem. Asian J. 2011, 6, 750.

[23] A. P. de Silva, J. Phys. Chem. Lett. 2011, 2, 2865.

[24] J. Andréasson, U. Pischel, Chem. Soc. Rev. 2015, 44, 1053. 
[25] F. Pu, J. S. Ren, X. G. Qu, Adv. Mater. 2014, 26, 5742.

[26] A. P. de Silva, Molecular logic-based computation, Royal Society of Chemistry, Cambridge 2016.

[27] Z. W. Dai, H. M. Leung, P. K. Lo, Small 2017, 13, 1602881.

[28] E. Katz, Molecular and supramolecular information processing: from molecular switches to logic systems, John Wiley \& Sons, New York 2013.

[29] F. Pu, J. Ren, X. Yang, X. Qu, Chem. Eur. J. 2011, 17, 9590.

[30] E. Katz, Enzyme-Based Computing Systems, John Wiley \& Sons, New York 2019.

[31] C. Zhang, J. Yang, J. Xu, Chinese Sci. Bull. 2011, 56, 3566.

[32] T. Gunnlaugsson, J. P. Leonard, Chem. Comm. 2005, 3114.

[33] S. J. Bradberry, J. P. Byrne, C. P. McCoy, T. Gunnlaugsson, Chem. Commun. 2015, 51, 16565.

[34] L. K. Truman, S. J. Bradberry, S. Comby, O. Kotova, T. Gunnlaugsson, Chem. Phys. Chem. 2017, 18, 1746.

[35] X. Y. Xu, B. Yan, Adv. Funct. Mater. 2017, 27, 1.

[36] M. Rodrigues, R. Piñol, G. Antorrena, C. D. S. Brites, N. J. O. Silva, J. L. Murillo, R. Cases, I. Díez, F. Palacio, N. Torras, J. A. Plaza, L. Pérez-García, L. D. Carlos, A. Millán, Adv. Funct. Mater. 2016, 26, 200.

[37] J. Andréasson, U. Pischel, S. D. Straight, T. A. Moore, A. L. Moore, D. Gust, J. Am. Chem. Soc. 2011, 133, 11641.

[38] J. Andréasson, S. D. Straight, G. Kodis, C. D. Park, M. Hambourger, M. Gervaldo, B. Albinsson, T. A. Moore, A. L. Moore, D. Gust, J. Am. Chem. Soc. 2006, 128, 16259.

[39] S. Silvi, E. C. Constable, C. E. Housecroft, J. E. Beves, E. L. Dunphy, M. Tomasulo, F. M. Raymo, A. Credi, Chem. Eur. J. 2009, 15, 178.

[40] M. Pars, C. C. Hofmann, K. Willinger, P. Bauer, M. Thelakkat, J. Kohler, Angew. Chem. Int. Edit. 2011, 50, 11405.

[41] M. Balter, S. M. Li, J. R. Nilsson, J. Andréasson, U. Pischel, J. Am. Chem. Soc. 2013, $135,10230$.

[42] S. Maji, S. Kumar, K. Sankaran, Spectrochim. Acta A 2015, 135, 405.

[43] C. D. S. Brites, P. P. Lima, N. J. O. Silva, A. Millán, V. S. Amaral, F. Palacio, L. D. Carlos, Adv. Mater. 2010, 22, 4499.

[44] C. D. S. Brites, P. P. Lima, N. J. O. Silva, A. Millán, V. S. Amaral, F. Palacio, L. D. Carlos, Front. Chem. 2013, 1, 9. 
[45] R. Piñol, C. D. S. Brites, R. Bustamante, A. Martínez, N. J. O. Silva, J. L. Murillo, R. Cases, J. Carrey, C. Estepa, C. Sosa, F. Palacio, L. D. Carlos, A. Millán, ACS Nano 2015, 9, 3134.

[46] C. D. S. Brites, P. P. Lima, L. D. Carlos, J. Lumin. 2016, 169 B, 497.

[47] C. D. S. Brites, M. C. Fuertes, P. C. Angelomé, E. D. Martínez, P. P. Lima, G. J. A. A. Soler-Illia, L. D. Carlos, Nano Lett. 2017, 17, 4746.

[48] M. Latva, H. Takalo, V.-M. Mukkala, C. Matachescu, J. C. Rodríguez-Ubis, J. Kankare, J. Lumin. 1997, 75, 149.

[49] A. Ródenas, D. Jaque, J. G. Solé, A. Speghini, M. Bettinelli, E. Cavalli, Phys. Rev. B 2006, 74, 035106.

[50] L. D. Carlos, V. D. Bermudez, V. S. Amaral, S. C. Nunes, N. J. O. Silva, R. A. S. Ferreira, J. Rocha, C. V. Santilli, D. Ostrovskii, Adv. Mater. 2007, 19, 341.

[51] D. V. G. L. N. Rao, F. J. Aranda, D. N. Rao, Z. Chen, J. A. Akkara, D. L. Kaplan, M. Nakashima, Opt. Commun. 1996, 127, 193.

[52] G. Y. Chen, Z. X. Guo, K. Chen, C. P. Zhang, J. G. Tian, Q. W. Song, Optik 2005, 116, 227.

[53] X. T. Liu, K. Wang, Z. Chang, Y. H. Zhang, J. L. Xu, Y. S. Zhao, X. H. Bu, Angew. Chem. Int. Edit. 2019, 58, 13890.

[54] A. Szukalski, M. Moffa, A. Camposeo, D. Pisignano, J. Mysliwiec, J. Mater. Chem. C 2019, 7, 170.

[55] M. Hatanaka, S. Yabushita, Theor. Chem. Acc. 2014, 133, 1517.

[56] B. Daly, T. S. Moody, A. J. M. Huxley, C. Y. Yao, B. Schazmann, A. Alves-Areias, J. F. Malone, H. Q. N. Gunaratne, P. Nockemann, A. P. de Silva, Nat. Commun. 2019, 10.

[57] G. de Ruiter, E. Tartakovsky, N. Oded, M. E. van der Boom, Angew. Chem. Int. Edit. 2010, 49, 169.

[58] U. Pischel, J. Andreasson, New J. Chem. 2010, 34, 2701.

[59] U. Pischel, Angew. Chem. Int. Edit. 2010, 49, 1356.

[60] W. Sun, C. Zhou, C. H. Xu, C. J. Fang, C. Zhang, Z. X. Li, C. H. Yan, Chem. Eur. J. 2008, 14,6342 .

[61] J. Andréasson, S. D. Straight, T. A. Moore, A. L. Moore, D. Gust, Chem. Eur. J. 2009, $15,3936$.

[62] Z. Q. Guo, W. H. Zhu, L. J. Shen, H. Tian, Angew. Chem. Int. Edit. 2007, 46, 5549.

[63] J. Andréasson, S. D. Straight, T. A. Moore, A. L. Moore, D. Gust, J. Am. Chem. Soc. 2008, 130, 11122 . 
[64] J. Andréasson, Y. Terazono, M. P. Eng, A. L. Moore, T. A. Moore, D. Gust, Dyes Pigments 2011, 89, 284.

[65] P. Remon, M. Hammarson, S. M. Li, A. Kahnt, U. Pischel, J. Andréasson, Chem. Eur. J. 2011, 17, 6492.

[66] J. Andréasson, U. Pischel, Isr. J. Chem. 2013, 53, 236.

[67] S. Buckhout-White, J. C. Claussen, J. S. Melinger, Z. Dunningham, M. G. Ancona, E. R. Goldman, I. L. Medintz, RSC Adv. 2014, 4, 48860.

[68] C. Ritchie, G. Vamvounis, H. Soleimaninejad, T. A. Smith, E. J. Bieske, V. Dryza, Phys. Chem. Chem. Phys. 2017, 19, 19984.

[69] J. F. C. B. Ramalho, S. F. H. Correia, L. S. Fu, L. L. F. Antonio, C. D. S. Brites, P. S. Andre, R. A. S. Ferreira, L. D. Carlos, Adv. Sci. 2019, 6, 1900950.

[70] C. D. S. Brites, P. P. Lima, N. J. O. Silva, A. Millán, V. S. Amaral, F. Palacio, L. D. Carlos, J. Lumin. 2013, 133, 230.

[71] D. Ananias, F. A. A. Paz, D. S. Yufit, L. D. Carlos, J. Rocha, J. Am. Chem. Soc. 2015, 137,3051 .

[72] C. Xia, C. Y. Yu, M. M. Cao, J. F. Xia, D. Y. Jiang, G. H. Zhou, D. F. Zhang, H. L. Li, Ceram. Int. 2018, 44, 21040.

[73] Y. J. Cui, W. F. Zou, R. J. Song, J. C. Yu, W. Q. Zhang, Y. Yang, G. D. Qian, Chem. Commun. 2014, 50, 719.

[74] A. M. Kaczmarek, D. Esquivel, B. Laforce, L. Vincze, P. Van Der Voort, F. J. RomeroSalguero, R. Van Deun, Luminescence 2018, 33, 567.

[75] H. Z. Wang, D. Zhao, Y. J. Cui, Y. Yang, G. D. Qian, J. Solid State Chem. 2017, 246, 341.

[76] S. H. Zheng, W. B. Chen, D. Z. Tan, J. J. Zhou, Q. B. Guo, W. Jiang, C. Xu, X. F. Liu, J. R. Qiu, Nanoscale 2014, 6, 5675.

[77] S. N. Zhao, L. J. Li, X. Z. Song, M. Zhu, Z. M. Hao, X. Meng, L. L. Wu, J. Feng, S. Y. Song, C. Wang, H. J. Zhang, Adv. Funct. Mater. 2015, 25, 1463.

[78] Y. H. Han, C. B. Tian, Q. H. Li, S. W. Du, J. Mater. Chem. C 2014, 2, 8065. 


\section{Figures}

$$
\mathbf{a}
$$
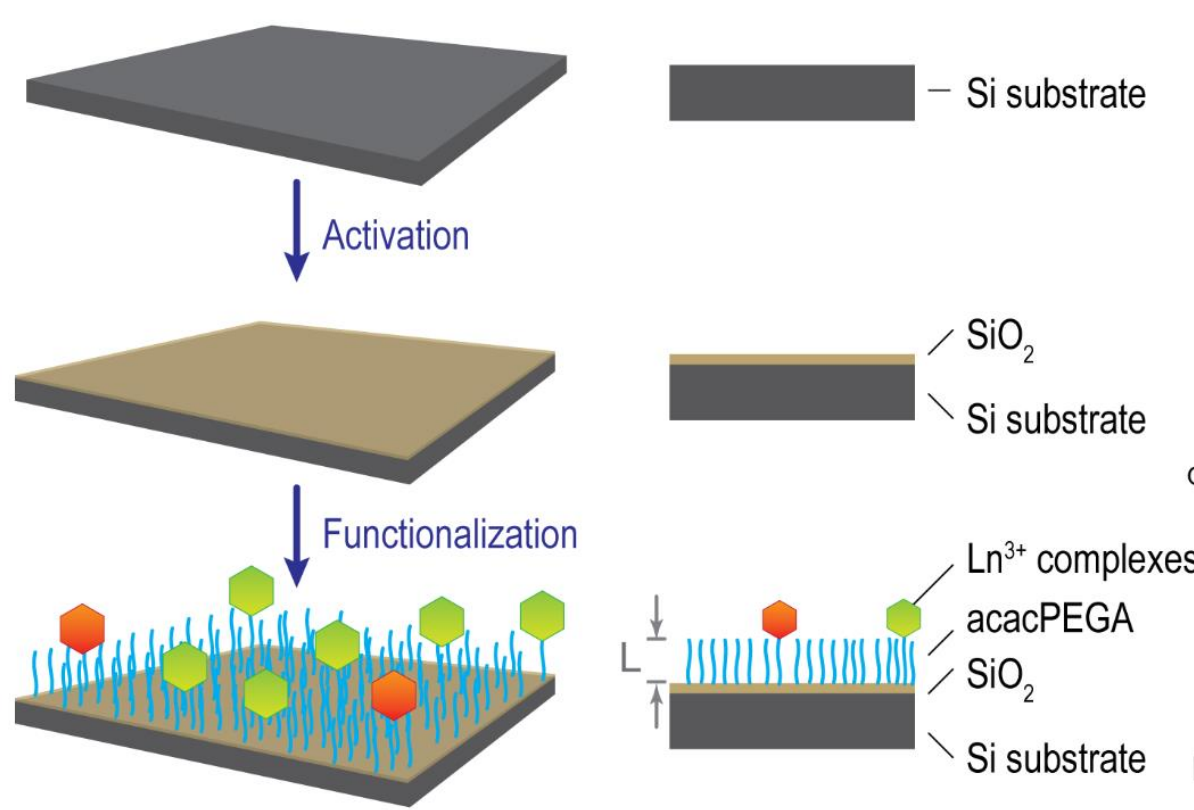

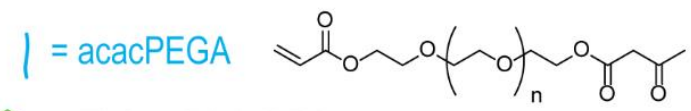

$=\mathrm{TbCl}_{3} \cdot 6 \mathrm{H}_{2} \mathrm{O} \mathrm{DPA}$

$=\mathrm{EuCl}_{3} \cdot 6 \mathrm{H}_{2} \mathrm{O} \mathrm{DPA}$
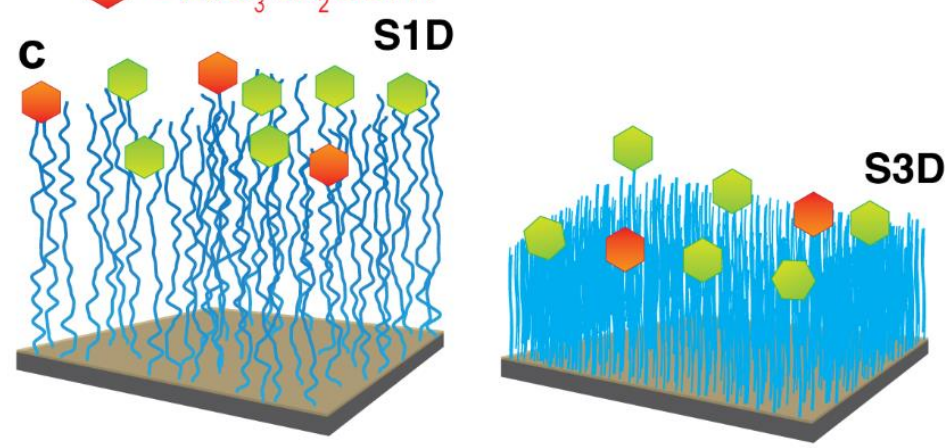

b
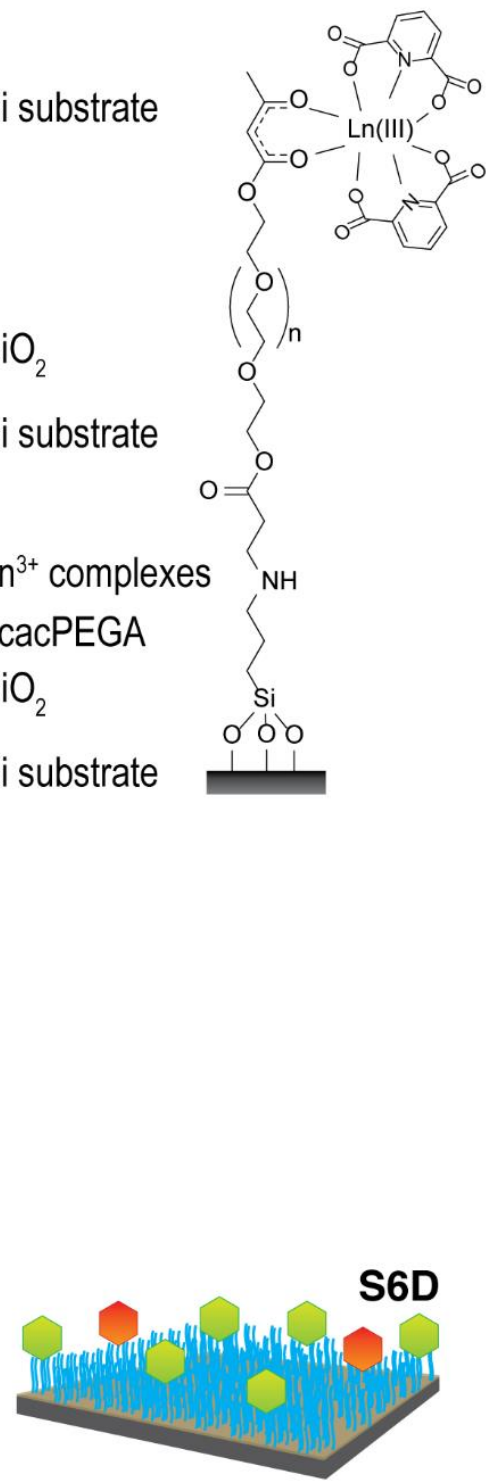

Figure 1. (a) Schematic diagram of $\mathrm{Si}$ surface functionalization with the self-assembled monolayers incorporating the $\mathrm{Ln}^{3+}$ ions showing the activation and functionalization steps. (b) The structural formula of monolayers functionalized with the $\mathrm{Ln}^{3+}$ ions. (c) Schematic representation of the S1D, S3D, and S6D samples. The details of the logic arrays structure and fabrication are summarized in Table 1. 

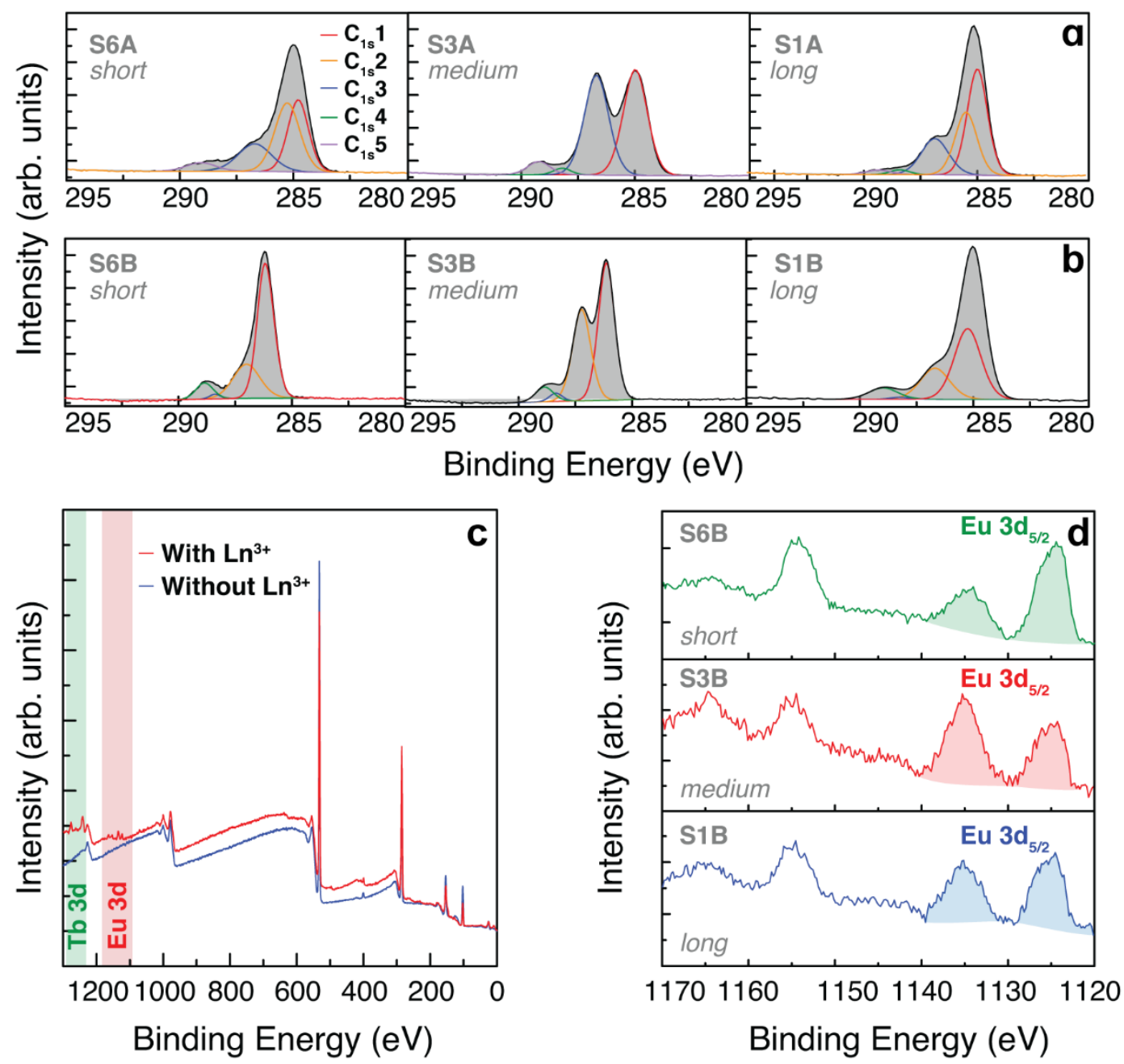

Figure 2. XPS spectra of the C 1s region for short (samples S6A, S6B) medium (S3A, S3B) and long (S1A, S1B) acacPEGA chains in samples (a) A series (b) B series (Table 1 of the main text). The carbon oxidation states in acacPEGA are $\mathrm{C}-\mathrm{C}\left(\mathrm{C}_{1 \mathrm{~s}} 1\right.$ and $\left.\mathrm{C}_{1 \mathrm{~s}} 2\right), \mathrm{C}-\mathrm{O}\left(\mathrm{C}_{1 \mathrm{~s}} 3\right)$, O-C$\mathrm{O} / \mathrm{C}=\mathrm{O}\left(\mathrm{C}_{1 \mathrm{~s}} 4\right)$ and $\mathrm{O}-\mathrm{C}=\mathrm{O}\left(\mathrm{C}_{1 \mathrm{~s}} 5\right)$. Remarkable differences are found in the $\mathrm{C}-\mathrm{O}$ signal depending on the length of the acacPEGA chain. Series $\mathrm{B}$ and $\mathrm{D}$, containing $\mathrm{Eu}^{3+}$ and $\mathrm{Tb}^{3+}$ ions, show the same behavior. (c) XPS spectra of S3A (before anchoring the complexes) and S3B (after anchoring the complexes). The $\mathrm{Tb} 3 \mathrm{~d}$ and Eu $3 \mathrm{~d}$ regions are highlighted to showing the differences resulting from the anchoring of the $\mathrm{Ln}^{3+}$ complexes. (d) Evolution of Eu $3 \mathrm{~d}$ core level with the length of the acacPEGA organic chain, for short (S6B), medium (S3B) and large (S1B) chains. 

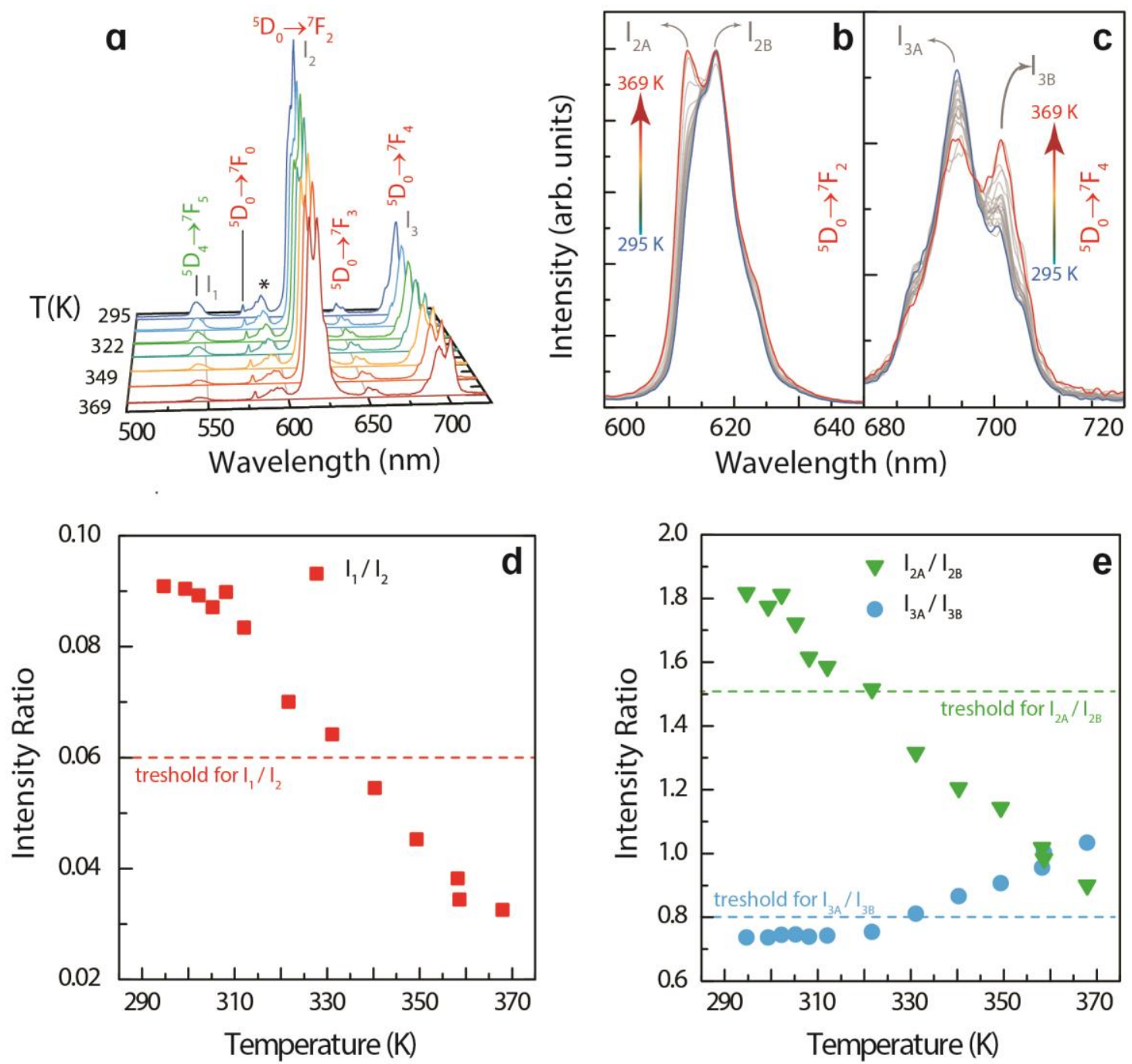

Figure 3. (a) Emission spectra of S6D in the 295-369 K range under $271 \mathrm{~nm}$ excitation. The $\mathrm{Tb}^{3+}$ and $\mathrm{Eu}^{3+}$ transitions are signed in green and red respectively. The asterisk marks the spectral region of overlap between the ${ }^{5} \mathrm{D}_{0} \rightarrow{ }^{7} \mathrm{~F}_{1}\left(\mathrm{Eu}^{3+}\right)$ and the ${ }^{5} \mathrm{D}_{4} \rightarrow{ }^{7} \mathrm{~F}_{4}\left(\mathrm{~Tb}^{3+}\right)$ transitions. The peak intensities $I_{1}, I_{2}$ and $I_{3}$ are also identified. Magnification of $(\mathbf{b}){ }^{5} \mathrm{D}_{0} \rightarrow{ }^{7} \mathrm{~F}_{2}$ (displaying the $I_{2 \mathrm{~A}}$ and $I_{2 \mathrm{~B}}$ components) and (c) ${ }^{5} \mathrm{D}_{0} \rightarrow{ }^{7} \mathrm{~F}_{4}$ (displaying the $I_{3 \mathrm{~A}}$ and $I_{3 \mathrm{~B}}$ components) transitions. The blue and red spectra correspond to the spectra measured at the lowest $(295 \mathrm{~K})$ and the highest $(369 \mathrm{~K})$ temperatures. Temperature dependence of the (d) $I_{1} / I_{2}$ intensity ratio and (e) $I_{2 \mathrm{~A}} / I_{2 \mathrm{~B}}$ and $I_{3 \mathrm{~A}} / I_{3 \mathrm{~B}}$ intensity ratios. The threshold values (above which the logical value of the ratio is " 1 " and below is " 0 ") are identified by the horizontal lines. 

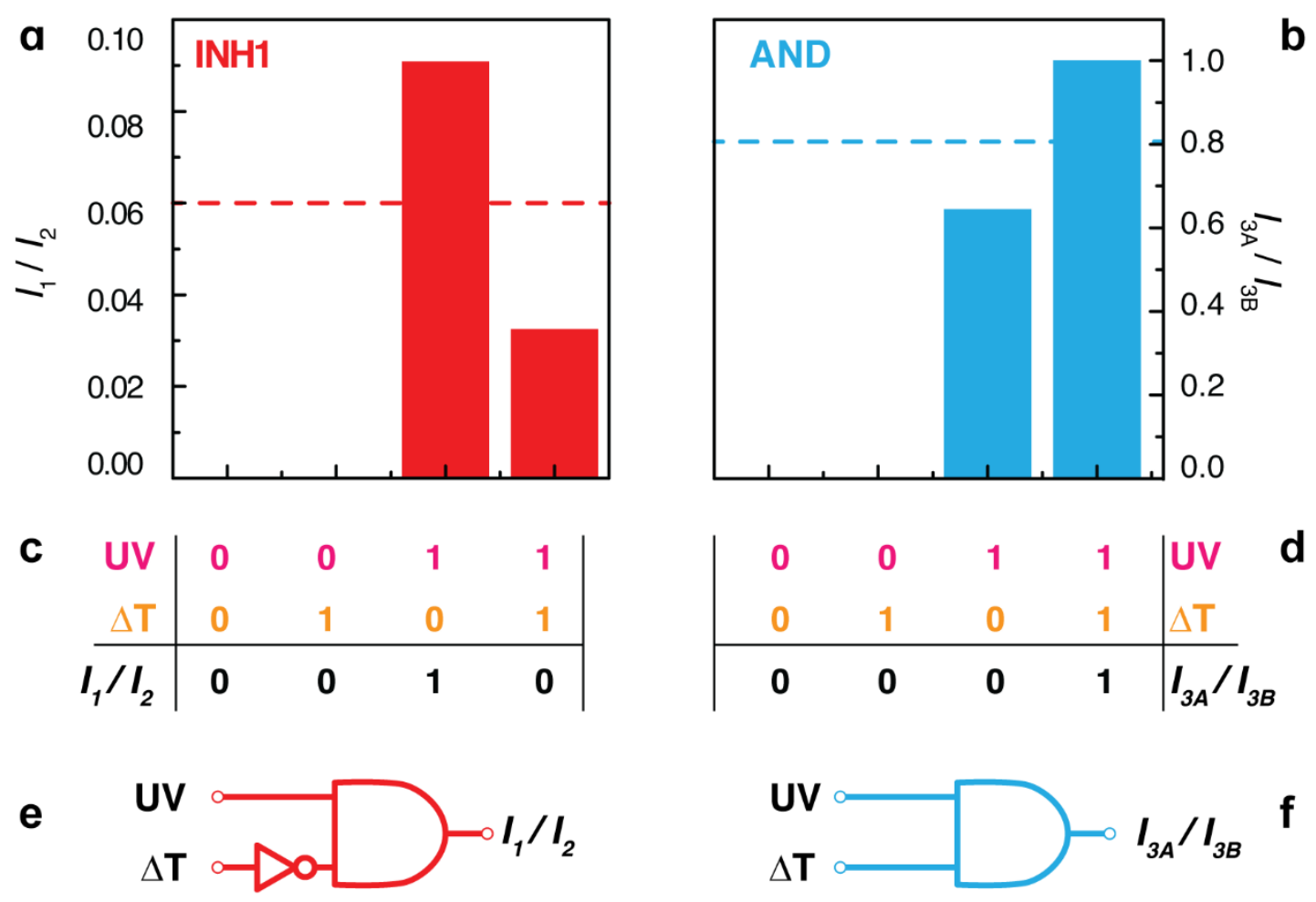

g

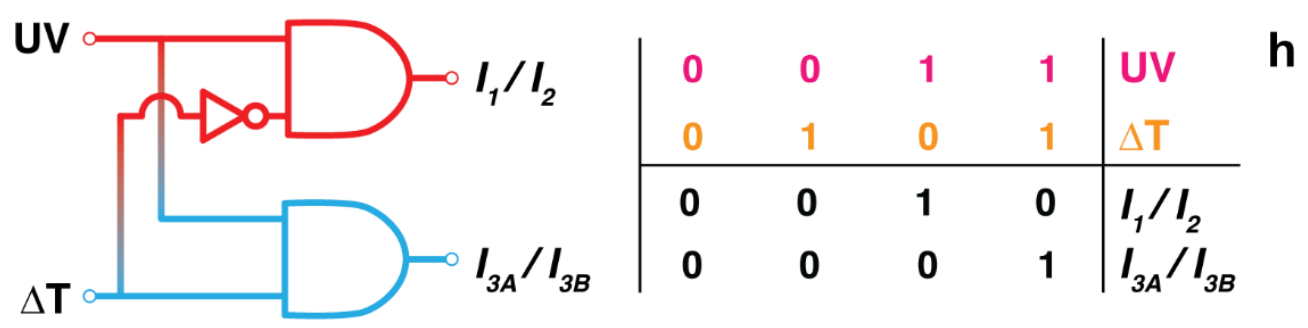

Figure 4. Bar plot of the (a) $I_{1} / I_{2}$ and (b) $I_{3 \mathrm{~A}} / I_{3 \mathrm{~B}}$ intensity ratios of S1D displaying a transfer function of inhibit 1 (INH1) and AND logical gates, respectively. The inputs used are the temperature increase (or heating, $\Delta \mathrm{T}$ ) and the $271 \mathrm{~nm}$ excitation (UV). The corresponding truth tables are presented in (c) and (d) and the schematic of the logical gates in (e) and (f). Combining the INH1 and AND logical gates the demultiplexer gate (DEMUX), in (g), is obtained. (h) The truth table for the DEMUX gate. 

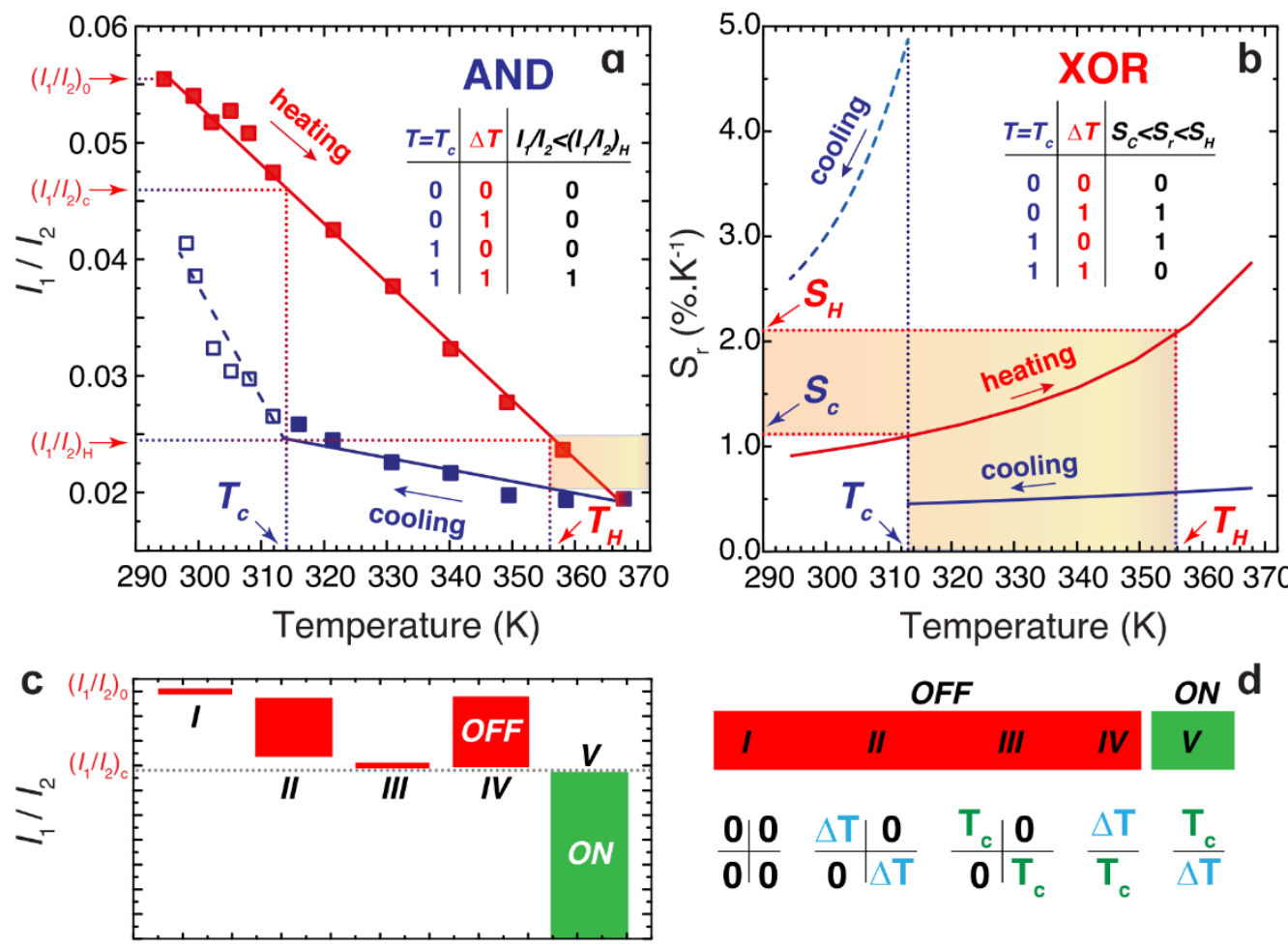

$$
\begin{array}{l|ll|lllll}
\mathbf{0} & \mathbf{0} & \Delta \mathrm{T} & \mathbf{0} & \mathrm{T}_{\mathrm{c}} & \mathbf{0} \\
\hline \mathbf{0} & \mathbf{0} & \mathbf{0} & \Delta \mathrm{T} & \mathbf{0} & \mathrm{T}_{\mathrm{c}} & \frac{\Delta \mathrm{T}}{\mathrm{T}_{\mathrm{c}}} & \frac{\mathrm{T}_{\mathrm{c}}}{\Delta \mathrm{T}}
\end{array}
$$

。

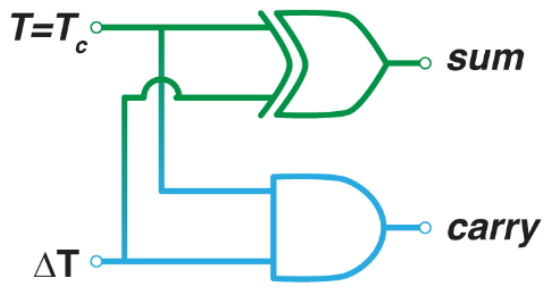

g

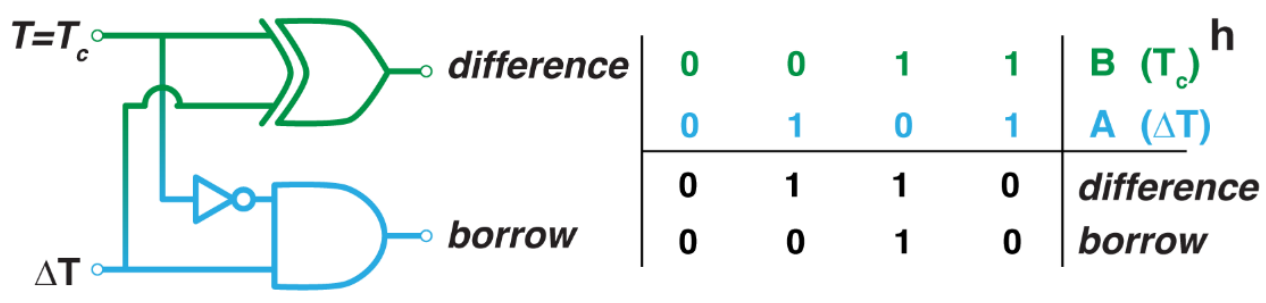

Figure 5. An illustrative plot of the (a) $I_{1} / I_{2}$ intensity ratio and (b) $S_{\mathrm{r}}$ of S1D. The vertical pointy lines mark the characteristic temperatures $T_{\mathrm{c}}$ and $T_{\mathrm{H}}$, whereas the horizontal ones indicate the corresponding values of $I_{1} / I_{2}$ (a) and $S_{\mathrm{r}}(\mathrm{b})$. The other lines in a) are the best fit to experimental data. In a and $b$, the shadowed regions mark the ranges in which the logical output is set as the logical value "1". (c) Bar plot of KEYPAD LOCK using the heating $(\Delta T)$ and $T_{\mathrm{c}}$ as inputs The ON state below the threshold (interrupted line) is only accessible when the inputs are applied in the correct sequence (first $T_{\mathrm{c}}$ and then $\Delta T$ ) leading to the lock opening, as shown in (d). Logic gates of arithmetic operations for (e) sum or (g) subtract two binary numbers. The corresponding truth tables are presented in (f) and (h). 


\section{Tables}

Table 1. The Si- functionalized surfaces are labeled accordingly to the molecular weight $\left(\mathrm{M}_{\mathrm{W}}\right.$, Da) of the PEG chains and the Michael addition reaction times ( $t=24$ hours or $t=48$ hours) employed in the thermal treatment of the $\mathrm{Ln}^{3+}$ doped and undoped samples. Details of the sample's synthesis are given in Supporting Information.

\begin{tabular}{cllll}
\hline \multirow{2}{*}{ PEG Mw } & \multicolumn{2}{c}{ without $\mathbf{L n}^{3+}$ ions } & \multicolumn{2}{c}{ with $\mathbf{L n}^{3+}$ ions } \\
& $24 h$ & $48 h$ & $24 h$ & $48 h$ \\
\hline 3000 & $S 1 A$ & $S 1 C$ & $S 1 B$ & $S 1 D$ \\
1000 & $S 2 A$ & $S 2 C$ & $S 2 B$ & $S 2 D$ \\
600 & $S 3 A$ & $S 3 C$ & $S 3 B$ & $S 3 D$ \\
400 & $S 4 A$ & $S 4 C$ & $S 4 B$ & $S 4 D$ \\
200 & $S 5 A$ & $S 5 C$ & $S 5 B$ & $S 5 D$ \\
100 & $S 6 A$ & $S 6 C$ & $S 6 B$ & $S 6 D$ \\
\hline
\end{tabular}


Table 2. Excitation wavelength $(\lambda, \mathrm{nm})$, temperature range $(\Delta T, \mathrm{~K})$, maximum relative sensitivity $\left(S_{\mathrm{m}}, \% \mathrm{~K}^{-1}\right)$, and corresponding temperature $\left(T_{\mathrm{m}}, \mathrm{K}\right)$ of illustrative $\mathrm{Ln}^{3+}$-based thermometers involving the intensity ratio of the ${ }^{5} \mathrm{D}_{4} \rightarrow{ }^{7} \mathrm{~F}_{5}$ and ${ }^{5} \mathrm{D}_{0} \rightarrow{ }^{7} \mathrm{~F}_{2}$ transitions.

\begin{tabular}{|c|c|c|c|c|c|}
\hline Material & $\lambda$ & $\Delta T$ & $S_{\mathrm{m}}$ & $T_{\mathrm{m}}$ & ref. \\
\hline $\begin{array}{l}\mathrm{Tb}^{3+} / \mathrm{Eu}^{3+} \text { co-doped organic-inorganic } \\
\text { di-ureasil hybrid }\end{array}$ & 325 & 298-333 & 7.1 & 298 & [47] \\
\hline $\mathrm{Eu}_{0.25} \mathrm{~Tb}_{0.75}(\mathrm{tfac})_{3} \cdot \mathrm{H}_{2} \mathrm{O}$ & 330 & 280-317 & 5.14 & 295 & [69] \\
\hline $\begin{array}{l}\mathrm{Tb}^{3+} / \mathrm{Eu}^{3+} \text { co-doped organic-inorganic } \\
\text { di-ureasil hybrid incorporating } \gamma-\mathrm{F}_{2} \mathrm{O}_{3} \\
\text { nanoparticles }\end{array}$ & 358 & $12-303$ & 3.0 & 125 & [70] \\
\hline S1D & 271 & 295-369 & 2.84 & 369 & \multirow{3}{*}{$\begin{array}{l}\text { This } \\
\text { work }\end{array}$} \\
\hline S3D & 271 & 295-369 & 2.23 & 369 & \\
\hline S6D & 271 & 295-369 & 2.27 & 369 & \\
\hline $\mathrm{Na}\left[\left(\mathrm{Gd}_{0.8} \mathrm{Eu}_{0.1} \mathrm{~Tb}_{0.1}\right) \mathrm{SiO}_{4}\right]$ & 483.5 & $12-450$ & 2.0 & 20 & [71] \\
\hline $\mathrm{Zn}_{4} \mathrm{O}(\mathrm{bdc})_{3}: \mathrm{Eu}^{3+} / \mathrm{Tb}^{3+}$ & 325 & $303-473$ & 1.8 & 473 & [72] \\
\hline $\mathrm{Tb}_{0.957} \mathrm{Eu}_{0.043} \mathrm{cpda}$ & 335 & $40-300$ & 1.77 & 250 & [73] \\
\hline $\begin{array}{l}\mathrm{Eu}^{3+} / \mathrm{Tb}^{3+} \text { dpa complexes in } \\
\text { acacPEGA }\end{array}$ & 280 & 296-338 & 1.43 & 323 & [36] \\
\hline $\begin{array}{l}\mathrm{Eu}^{3+} / \mathrm{Tb}^{3+} \text { co-doped mesoporous } \\
\text { silica decorated with dppz ligands }\end{array}$ & 295 & $10-360$ & 1.32 & 260 & [74] \\
\hline $\begin{array}{l}\mathrm{Eu}_{0.37} \mathrm{~Tb}_{0.63} \text {-btc metal organic } \\
\text { framework }\end{array}$ & 296 & $313-473$ & 0.68 & 313 & [75] \\
\hline $\begin{array}{l}\mathrm{NaGdF}_{4}: \mathrm{Yb}^{3+} / \mathrm{Tm}^{3+} @ \mathrm{~Tb}^{3+} / \mathrm{Eu}^{3+} \\
\text { nanoparticles }\end{array}$ & 980 & $125-300$ & 0.49 & 300 & [76] \\
\hline $\mathrm{Na}\left[\left(\mathrm{Gd}_{0.8} \mathrm{Eu}_{0.1} \mathrm{~Tb}_{0.1}\right) \mathrm{SiO}_{4}\right]$ & 483.5 & $10-425$ & 0.3 & 300 & [71] \\
\hline $\mathrm{Tb}_{0.8} \mathrm{Eu}_{0.2} \mathrm{~L}$ & 322 & $40-300$ & 0.15 & 300 & [77] \\
\hline$\left[\mathrm{Tb}_{0.3} \mathrm{Eu}_{0.7}(\mathrm{D}-\mathrm{cam})(\mathrm{Himdc})_{2} \cdot\left(\mathrm{H}_{2} \mathrm{O}\right)_{2}\right]_{3}$ & 277 & $100-450$ & 0.11 & 450 & [78] \\
\hline
\end{tabular}

Htfac=1,1,1-trifluoro-2,4-pentanedione; $\mathrm{H}_{2} \mathrm{bdc}=1$,4-benzenedicarboxylic acid; $\mathrm{H}_{2} \mathrm{dpa}=2,6$ pyridinedicarboxylic acid; $\mathrm{H}_{3} \mathrm{cpda}=5$-(4-carboxyphenyl)-2,6-pyridinedicarboxylic acid; dppz=dipyridyl-pyridazine; $\quad \mathrm{H}_{3}$ btc $=1,3,5$-benzenetricarboxylic $\quad$ acid; $\quad \mathrm{H}_{2} \mathrm{~L}=1,3$-bis(4carboxyphenyl)imidazolium; $\quad \mathrm{D}-\mathrm{H}_{2} \mathrm{cam}=\mathrm{D}$-camphoric $\quad$ acid; $\quad \mathrm{H}_{3} \mathrm{imdc}=4.5$-imidazole dicarboxylic acid. 


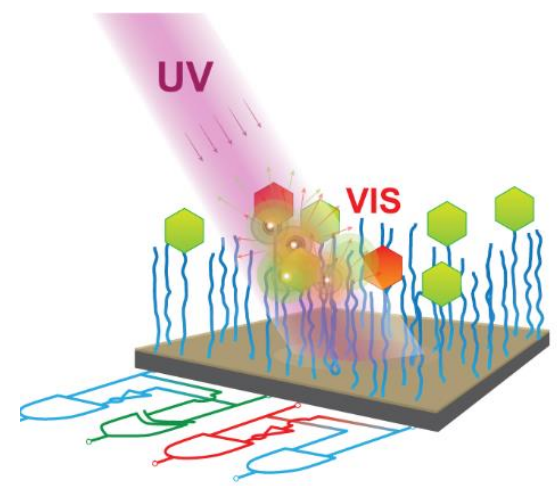

Monolithic silicon-based structures incorporating $\mathrm{Ln}^{3+}$ complexes in self-assembled polymeric layers are developed for mimicking molecular logic and computing. Elementary logic gates (AND, INH and DEMUX), sequential logic (KEYPAD LOCK), and arithmetic operations (HALF ADDER and HALF SUBTRACTOR) are demonstrated here for the first time in non-wet conditions.

Keywords lanthanide, luminescence, molecular logic, computing, physical input

M. A. Hernández-Rodríguez, C. D. S. Brites, * G. Antorrena, R. Piñol, R. Cases, L. Pérez-García, M. Rodrigues, J. A. Plaza, N. Torras, I. Díez, A. Millán, L. D. Carlos*

Lanthanide Luminescence to Mimic Molecular Logic and Computing through Physical Inputs 